\title{
Characterization of blinking dynamics in quantum dot ensembles using image correlation spectroscopy
}

\author{
Alexia I. Bachir \\ Department of Chemistry, McGill University, Montreal, Quebec H3A 2K6, Canada \\ Nela Durisic, Benedict Hebert, and Peter Grütter \\ Department of Physics, McGill University, Montreal, Quebec H3A 2T8, Canada \\ Paul W. Wiseman ${ }^{\text {a) }}$ \\ Department of Chemistry, McGill University, Montreal, Quebec H3A 2K6, Canada \\ and Department of Physics, McGill University, Montreal, Quebec H3A 2T8, Canada
}

(Received 25 August 2005; accepted 24 January 2006; published online 16 March 2006)

\begin{abstract}
Quantum dots (QDs) are being increasingly applied as luminescent labels in optical studies for biophysical and cell biological applications due to their unique spectroscopic properties. However, their fluorescence "blinking" characteristics that follow power law statistics make it difficult to use QDs in some quantitative biophysical applications. We present image correlation spectroscopy (ICS) in combination with total internal reflection fluorescence microscopy as a tool to characterize blinking dynamics in QDs. We show that the rate of decay of the ICS measured ensemble correlation function reflects variation in blinking dynamics and can be used to distinguish different blinking distribution regimes. To test and confirm our hypothesis, we also analyze image time series simulations of ensembles of point emitters with set blinking statistics. We show that optimization of the temporal sampling and the number of QDs sampled is essential for detecting changes in blinking dynamics with ICS. We propose that this experimental characterization of the QD blinking statistics can actually serve as a sensitive reporter for certain quantitative biological applications. (C) 2006 American Institute of Physics. [DOI: 10.1063/1.2175470]
\end{abstract}

\section{INTRODUCTION}

Semiconductor (CdSe) ZnS quantum dots (QDs) are luminescent and highly photostable nanocrystals with dimensions smaller than about $10 \mathrm{~nm} .{ }^{1}$ Their band gap increases when the size of the particle decreases due to quantum confinement effects ${ }^{2}$ which produces distinctive size dependent photophysical properties that have received much attention recently. ${ }^{3,4}$ Their potential for biophysical studies was confirmed in late 1998 in experimental applications that demonstrated that semiconductor nanocrystals could be made biocompatible and used as photostable fluorescent probes to study biomolecules within living cells. ${ }^{5,6}$ However, QDs also exhibit fluorescence emission intermittency (so-called QD blinking), ${ }^{7}$ and this photophysical property limits quantitative application of these nanoparticles for certain biophysical applications. The blinking cannot easily be blocked or attenuated under cell physiological conditions, and the variations in fluorescence emission complicate the interpretation of analytical techniques, such as correlation spectroscopy, that rely on intensity as a reporter of particle mobility. In certain techniques, the blinking can fundamentally limit the statistics of individual measurements, such as in fluorescence microscopy based single particle tracking (SPT), where a switch to a dark "off" state leads to an abrupt termination of tracking of the QD tagged macromolecule of interest. ${ }^{8} \mathrm{Nev}-$ ertheless, trajectories in which QDs remain "on" for long

\footnotetext{
a) Author to whom correspondence should be addressed; electronic mail: paul.wiseman@mcgill.ca
}

durations can be selected to ensure accurate and precise determination of mobility coefficients by SPT. On the other hand, in correlation spectroscopy studies of diffusion, the measurement is ultimately based on statistical analysis of fluorescence fluctuations, so systematic errors cannot be avoided if blinking is not appropriately modeled. ${ }^{9}$ Mathematical models have been developed that incorporate the effects of probe blinking into correlation spectroscopy theory. These have been applied in fluctuation spectroscopy experiments that use certain organic dyes and fluorescent proteins which exhibit single exponential blinking kinetics with a single characteristic fluctuation time scale. ${ }^{10,11}$ However, the major challenge in adapting the correlation spectroscopy methods for QD blinking is that intermittency periods follow power law statistics with contributions over many orders of magnitude in time, ${ }^{12}$ so the single state blinking models cannot be applied.

To date, characterization of blinking in QDs has relied on the measurement of the probability distribution function through histogram binning of the "on" and "off" durations for fluorescence intensity time traces recorded from single QDs. ${ }^{7,12,13}$ Several theoretical studies have derived mathematical models for the correlation function decay of blinking phenomena with power law statistics, ${ }^{14,15}$ but these have not been applied in confocal microscopy or other highly sensitive optical experimental studies. Although there have been a large number of studies on blinking dynamics in quantum dots, a full understanding of how it affects fluorescence correlation measurements is still lacking.

In this total internal reflection fluorescence-image corre- 

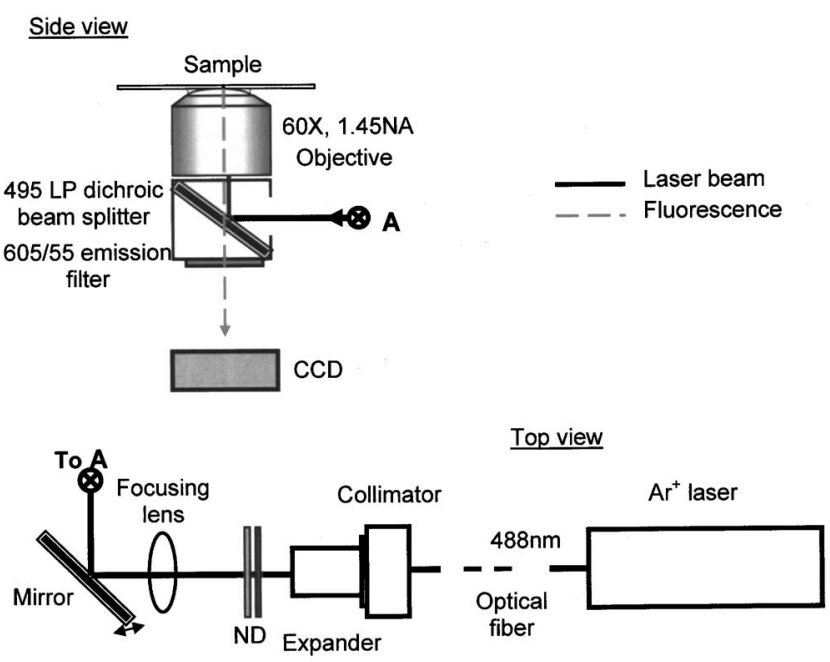

FIG. 1. Experimental setup scheme.

lation spectroscopy (TIRF-ICS) time series study, we characterize the blinking of an ensemble of spatially resolved immobile QDs on a glass substrate to restrict the measured fluctuations to those from the QD intermittency. We determine the ensemble intensity correlation function of the "blinking" process at various laser excitation powers. We show that the improved sampling inherent in the combined spatial and temporal image correlation analysis allows us to characterize QD blinking statistics from relatively short $(<1 \mathrm{~min})$ image time series. We verify that the variation in blinking statistics is indeed reflected in the measured ensemble correlation function by analyzing simulations of spatially distributed point emitters with set blinking power laws. As well, we determine the limits of temporal sampling and the necessary number of QDs imaged that are needed for accurate and precise measurement of the blinking statistics using ICS.

\section{EXPERIMENTAL METHOD}

We used streptavidin functionalized (CdSe) ZnS quantum dots (QD605-streptavidin, Quantum Dot Corp., Hayward, CA) with emission wavelength centered at $605 \mathrm{~nm}$. The QD samples were sonicated for $15 \mathrm{~min}$ and then deposited on freshly cleaned coverslips prior to use. Microscope coverslips (Fisher Scientific) were rinsed with ethanol, boiled in 1:1 mixture of chloroform and ethanol for $15 \mathrm{~min}$, then rinsed again with copious amounts of ethanol and de-ionized water, and blown dry under nitrogen gas. Solutions of the QDs in water were deposited immediately on the dried glass coverslips. Excess QD solution was removed by nitrogen flow shortly after deposition to prevent formation of aggregates on the surface of the glass due to cohesion forces during the drying process.

We used objective-type total internal reflection fluorescence microscopy (TIRFM) for time series imaging of the QDs on the glass substrates. The TIRFM apparatus, shown in Fig. 1, was custom built around a Zeiss Axiovert S100TV inverted microscope using an optical setup similar to that of Paige et al. ${ }^{16}$ The $488 \mathrm{~nm}$ line from a cw $\mathrm{Ar}^{+}$laser (Melles Griot 35 LAP 431) was used for evanescent excitation through a Zeiss Planapo $100 \times 1.45$ numerical aperture (NA) objective lens. The excitation power was attenuated using neutral density (ND) filters. Fluorescence from the sample plane was filtered with an emission filter centered at $605 \mathrm{~nm}$ with bandwidth of $55 \mathrm{~nm}$ (Croma Technology, Rockingham, VT). Image time series were recorded using an intensified PentaMax charge-coupled device (CCD) camera (Princeton Instruments, Trenton, NJ) with $50 \mathrm{~ms}$ integration time and 25 ms readout time so that the time between subsequent images is $75 \mathrm{~ms}$. During the collection time and during the readout time the dots are blinking. Due to the self-similarity property of Levy statistics, the information, which is not accounted for during the readout time, does not alter the "on" and "off" probability distribution exponents. To confirm that, we conducted experiments with integration to readout time ratios varying from 0.4 to 28 and obtained similar results (data not shown).

Computer simulations of image time series of ensembles of spatially resolved blinking point sources were run using programs written in Interactive Data Language (IDL 6.0, RSI Colorado). Random pixel positions were selected for the point source particles according to a set particle number variable. Each pixel was then convolved with a two-dimensional (2D) Gaussian function of $0.3 \mu \mathrm{m} e^{-2}$ radius to simulate the spatial form of the detected point fluorescence emission profile due to the point spread function (PSF) of the optics. The "on" and "off" time durations were governed by inverse power laws with set variable exponents that were produced using the transformation method of uniformly distributed random numbers: ${ }^{17}$

$$
P\left(t_{\mathrm{on} / \mathrm{off}}\right) \propto \frac{1}{t_{\mathrm{on} / \mathrm{off}}^{m_{\mathrm{off}}}} .
$$

Since the power law decays for exponents greater than 1 are nonintegrable near the origin, we chose the time step between images $\Delta t$ as the minimum dwell time of the "on" or "off" state while infinitely long "on" and "off" times were allowed. The "off" time distribution exponent $m_{\text {off }}$ was set to 1.5 , while the "on" time distribution exponent $m_{\text {on }}$ was varied from 1.5 to 1.9. The choice of exponents was based on the observed experimental values for capped $(\mathrm{CdSe}) \mathrm{ZnS}$ quantum dots. ${ }^{13,18,19} \mathrm{CCD}$ camera integration and readout time were not simulated.

The discrete normalized ensemble intensity autocorrelation function for an image time series was calculated as follows: $:^{20}$

$$
r(s)=\frac{1}{N-s} \sum_{q=1}^{N-s} \frac{1}{M_{1} M_{2}} \sum_{x=1}^{M_{1}} \sum_{y=1}^{M_{2}} \frac{i(x, y, q) i(x, y, q+s)}{\langle i\rangle_{c}\langle i\rangle_{c+s}},
$$

where $s$ is the discrete image lag variable and is evaluated in integer steps from $s=0$ to $N-1, M_{1}$ and $M_{2}$ are the number of pixels in the horizontal and vertical dimensions of a rectangular planar analyzed subregion of interest in the image, and $N$ is the total number of images in the time series. The $\langle i\rangle_{c(\text { or } c+s)}$ indicates spatial averaging of the intensity across the subregion in image $c$ or $c+s$, respectively. This discrete approximation to the temporal lag autocorrelation function $[r(\tau)]$ was obtained by simply converting the discrete image 
lag independent variable $s$ to a discrete time lag: $\tau=s \delta t$ where $\delta t$ is the time step between sequential image frames.

\section{RESULTS AND DISCUSSION}

A TIRF image of immobilized and spatially resolved streptavidin functionalized capped $(\mathrm{CdSe}) \mathrm{ZnS}$ QDs is presented in Fig. 2(a). The fluorescence intensity imaged on the CCD from spatially distributed QDs on the substrate follows an extended 2D Gaussian intensity profile characteristic of evanescent field illumination. The normalized ensemble intensity time correlation functions of blinking QDs were calculated from time series stacks of $15 \times 15 \mu \mathrm{m}^{2}$ area, selected from the central region of the image series as highlighted in Fig. 2(a). In this region, the extended Gaussian illumination profile decays by less than $10 \%$ in intensity at the periphery as determined from the signal obtained from fluorescent dye uniformly spread on a glass coverslip. Single pixel intensity traces selected from the centroids of the highlighted QDs from Fig. 2(a) are presented in Fig. 2(b) and the corresponding single trace intensity autocorrelation functions in Fig. 2(c). Figure 2 illustrates some of the problems inherent in single dot intensity correlation analysis for the case when there is limited time sampling. Clearly, for dots A and B, the autocorrelation decays reflect the step-function-like behavior of the intensity traces and effective under sampling of the distributions. The autocorrelation functions are disproportionately affected by the long "on" time in case A and the long "off" time in case B. It is not possible to accurately fit such autocorrelation functions and they would have to be excluded from the analysis. Quantum dot $\mathrm{C}$ produces a signal that fluctuates rapidly in time, so long time correlation is absent. In this case, the autocorrelation function decays too rapidly as no long time blinking events are sampled. The autocorrelation functions for dots D and E can be accurately fitted and analyzed as the parent intensity time traces contain a (random) sampling of blinking events over a range of time scales. We find that in the cases where time sampling is necessarily limited, careful selection of intensity traces must be exercised to obtain accurate results from intensity autocorrelation analysis of single QDs. This result is not surprising considering the long integration times $(>10 \mathrm{~min})$ employed in previous single dot histogram studies of QD blinking properties. ${ }^{12,13}$ However, it is important to note that this type of data selection is difficult to perform without introducing experimental bias into the analysis.

In contrast, we find that for these inherently short sampling times, the ensemble ICS analysis can be performed without excluding any of the emitters, and we obtained results that accurately reflect the underlying distribution of "on" and "off" blinking times. In the ensemble analysis of streptavidin functionalized $(\mathrm{CdSe}) \mathrm{ZnS}$ QDs on a glass substrate, the observed normalized intensity time autocorrelation functions are slowly decaying power laws with no single characteristic decay time (Fig. 3). The same type of decay was observed for the intensity time autocorrelation functions of nonfunctionalized single capped $(\mathrm{CdSe}) \mathrm{ZnS}$ quantum dots and interpreted qualitatively based on the fact that in capped $(\mathrm{CdSe}) \mathrm{ZnS}$ quantum dots, the "on" and "off" time durations

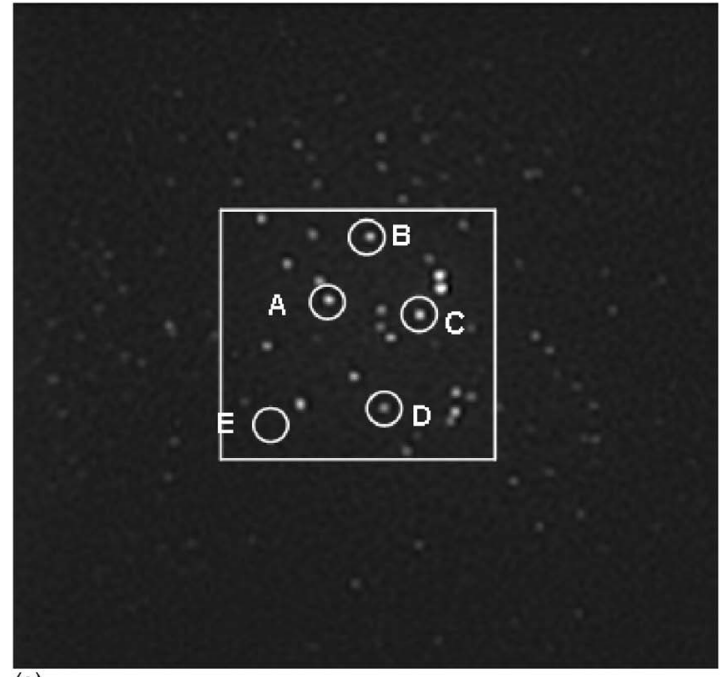

(a)

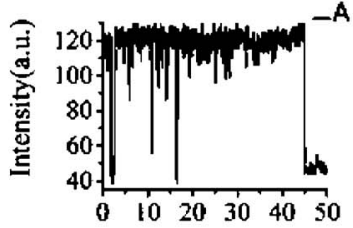

$\mathrm{t}(\mathrm{s})$

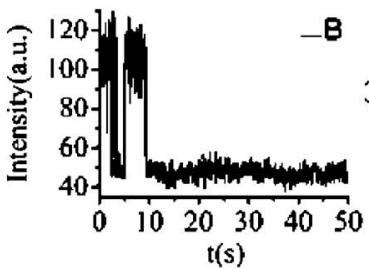

t(s)
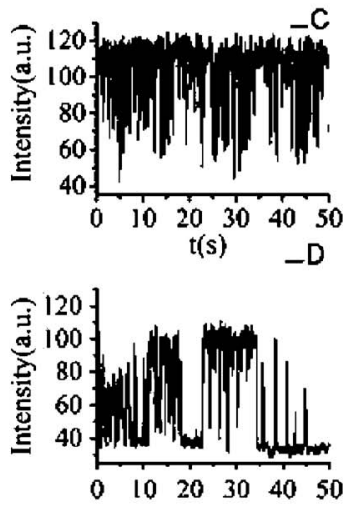

$\mathrm{t}(\mathrm{s})$

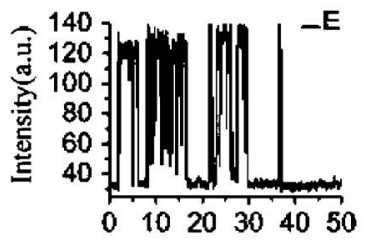

(b)

$\mathrm{t}(\mathrm{s})$

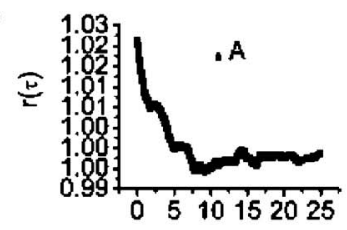

$\tau(s)$
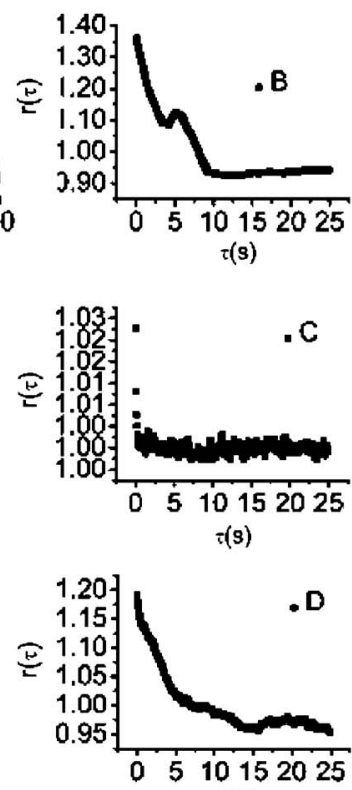

$\tau(\mathbf{s})$

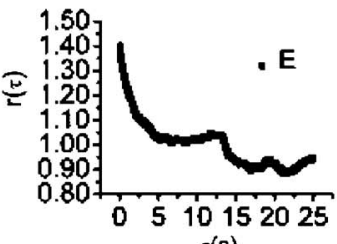

$\tau(s)$
FIG. 2. (Color online (a) TIRFM image of streptavidin functinonalized (Cd$\mathrm{Se}) \mathrm{ZnS}$ QDs immobilized on a glass coverslip. (b) Intensity time traces of selected individual QDs. (c) Corresponding normalized intensity time correlation function of single QDs' intensity time trace presented in (b)

follow inverse power law distributions. "21 "On" times on the order of the total measurement time are likely to occur for a fraction of quantum dots. They will appear as long range 


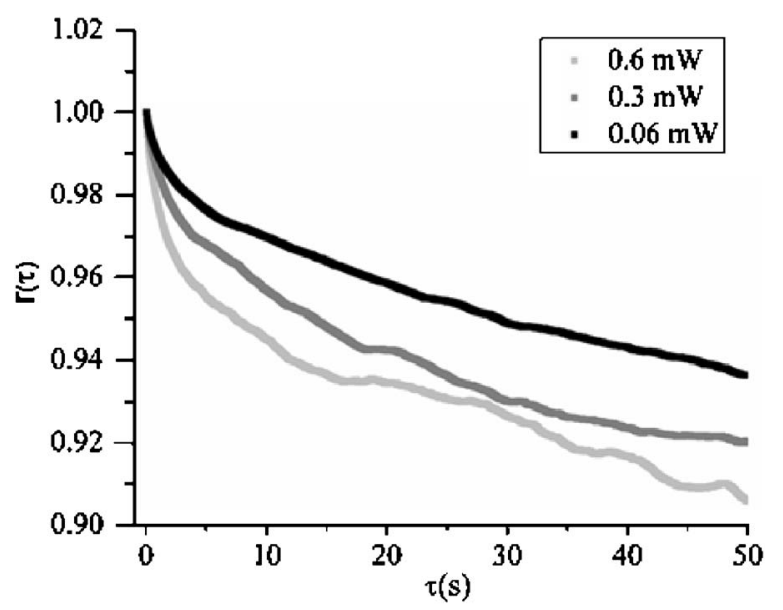

FIG. 3. Normalized intensity time correlation functions for various laser excitation powers as calculated from 2000 frame image time series with $50 \mathrm{~ms} /$ frame integration time for blinking streptavidin functionalized $(\mathrm{Cd}-$ $\mathrm{Se}) \mathrm{ZnS}$ QDs on a glass substrate. $0.06 \mathrm{~mW}(\boldsymbol{\square}), 0.3 \mathrm{~mW}(\boldsymbol{\square})$, and $0.6 \mathrm{~mW}$ $(\square)$ excitation laser powers. Intensity correlation functions are normalized to 1 for comparison.

correlations in the correlation function, causing it to decay slowly over the time scale of the measurement, as is observed in Fig. 3. The intensity autocorrelation functions presented in Fig. 3 show an increased rate of decay at higher excitation laser intensities as a result of the shorter "on" events observed at high laser powers. ${ }^{22}$

To quantify the rate of decay of the ensemble time autocorrelation functions, we fit using a three parameter power law:

$$
r(\tau)=A-B \tau^{\alpha}
$$

where $A, B$, and $\alpha$ are the fitting parameters. We use the fitting exponent $\alpha$ as a measure of the rate of decay of the correlation function since it reflects the underlying "on" and "off" time distribution exponents. The coefficients $A$ and $B$ depend on the underlying blinking statistics as well as the average "on" time of the ensemble of QDs. An explicit dependence of these parameters on the fitting exponents has been proposed by Verberk and Orrit who model the nonnormalized intensity correlation function of capped $(\mathrm{Cd}-$ $\mathrm{Se}) \mathrm{ZnS}$ QDs. ${ }^{23}$ It is worth noting that their model applies to the intensity time correlation function of a single QD, whereas our correlation functions are calculated for an ensemble of QDs. The distinction is of relevance given the nonstationary and nonergodic properties of power law distributions, where the average "on" time of an ensemble of blinking QDs is time dependent and does not correspond to the average "on" time of a single QD calculated with infinite collection time. These properties greatly complicate the derivation of closed form analytical expressions for theoretical single dot and ensemble correlation functions. Margolin and Barkai have presented full theoretical treatments of the nonergodic properties of time averaged correlation functions in single QDs (Ref. 15) and the aging properties of correlation function in ensembles of QDs. ${ }^{14}$ However, our experimental results show that for the sampling regimes we explored, the ensemble intensity autocorrelation function decays of protein

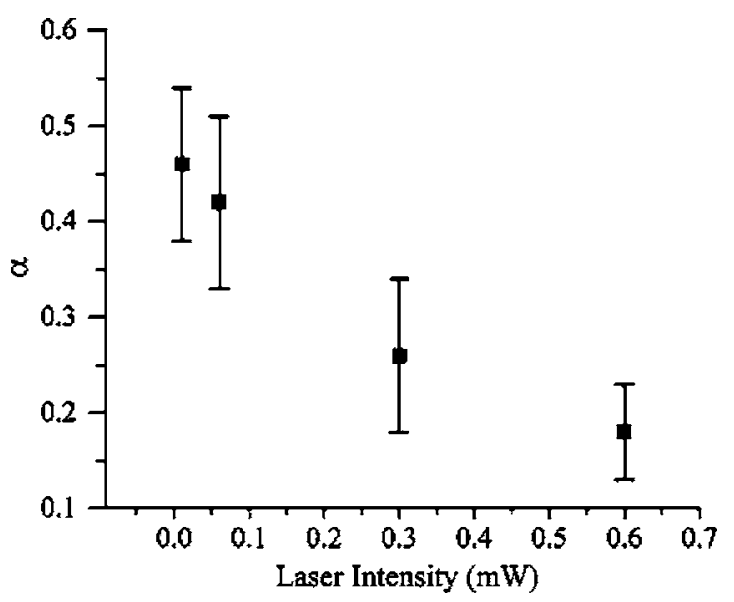

FIG. 4. Plot of $\alpha$ as a function of laser power. Each value is an average from 15 measurements. The error bars are standard deviations.

functionalized capped (CdSe)ZnS QDs are well modeled by the simple three parameter power law.

A plot of the mean recovered $\alpha$ exponent as a function of laser power is presented in Fig. 4. We observe a decrease in $\alpha$ from $0.46 \pm 0.08$ at $0.01 \mathrm{~mW}$ laser power to $0.18 \pm 0.05$ at $0.6 \mathrm{~mW}$. We also found that the ensemble autocorrelation function cannot be properly fitted by the three parameter power law [Eq. (3)] outside of this range of excitation power. At powers lower than $0.06 \mathrm{~mW}$, longer "on" events dominate and remain correlated over the time scale of the measurement, resulting in a correlation function which barely decays. At powers higher than $0.6 \mathrm{~mW}$, the opposite effect is observed, the correlation function rapidly decays, and the autocorrelation function cannot be modeled by a slowly decaying power law. The latter case is a possible explanation as to why the correlation functions of single CdSe QDs in the study reported by Kobitski et al. fail to fit to the power law decay model of Verberk and Orrit. ${ }^{24}$ In that study the absence of a passivating $\mathrm{ZnS}$ shell reduced the observation of long "on" time events. It is important to stress that the measured $\alpha$ exponent values reported at different laser intensities and the autocorrelation function decay model may differ depending on the type of functionalization of (CdSe)ZnS QDs.

We compared our experimental results to computer simulations to model what is expected for pure power law decays and to probe the precision and detection limits of the ICS method. By performing ICS analysis on simulated image time series of point emitters blinking according to set power law distributions, we tested the sensitivity of the method as a function of temporal sampling and of the number of emitters sampled.

The normalized intensity time autocorrelation functions calculated from the simulated image time series decay as slow power laws (see Fig. 5) as is expected based on the set distribution parameters for the simulation. A qualitative comparison of Figs. 3 and 5 shows that the experimental autocorrelation decays compare well with the autocorrelation functions from the simulations. Changes in the set "on" time distribution exponents for the simulations are directly reflected by the changes in the rate of decay of the calculated ensemble autocorrelation functions. In distributions with 


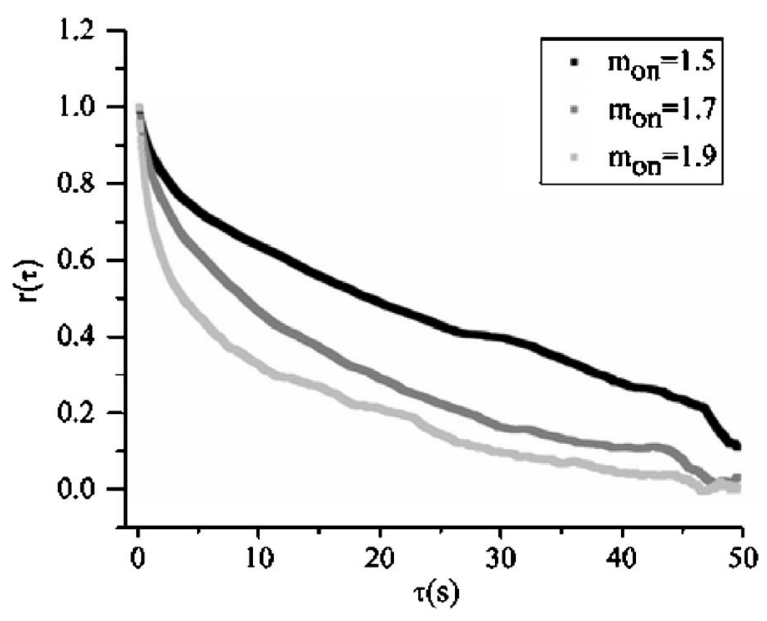

FIG. 5. Normalized intensity correlation functions calculated from 1000 frame simulated image series with an image time step of $0.05 \mathrm{~s}$. Each image consists of 300 point emitters. The "off" time probability distribution exponent is 1.5 for all simulated image series. The "on" time distribution exponents are $1.5(\mathbf{\square}), 1.7(\mathbf{\square})$, and $1.9(\square)$ respectively.

larger exponents, short "on" times dominate, causing the correlation function to decay more rapidly, an effect similar to what is observed at high laser excitation intensities. We use Eq. (3) to fit the ensemble correlation functions and recover the best fit exponent parameter $\alpha$.

Table I presents a summary of the exponent $\alpha$ determined by the ensemble correlation analysis from image time series simulations with 1000 frames, $m_{\text {on }}$ values ranging from 1.5 to 1.9 , and for different numbers of blinking point emitters within the ensemble. The recovered $\alpha$ values from the simulations show that the image correlation method is sensitive to changes in the power law distribution exponents, but the sensitivity and precision of the measurement are dependent on the total number of emitters sampled. For example, we observe an average 50\% decrease in the standard deviation of the mean recovered $\alpha$ exponent when the number of point emitters within the ensemble increases from 50 to 500. Also, ensembles containing more point emitters are more sensitive to variations in underlying blinking dynamics due to the improved sensitivity and precision of the recovered $\alpha$ exponent.

We found that an increase in temporal sampling improves the sensitivity of the ICS method and can be used to improve the measurement resolution for ensembles containing small numbers of point emitters. The results in Table II show that for a system of 150 point emitters, increasing the temporal sampling from 100 to 10000 image frames results in an average $14 \%$ decrease in the standard deviation on the mean recovered $\alpha$ exponent. In the sampling regimes we investigated, the temporal sampling has a much smaller influence on the precision of the measurements. In comparing the results for ensembles containing 800 and 150 point emitters in Tables I and II, we note that a more than an order of magnitude increase in temporal sampling is required to achieve the same sensitivity of resolution of the measured $\alpha$ value as is achieved with a fivefold increase in the number of point emitters sampled. For small ensembles containing less than 50 blinking point emitters, we increased the temporal sampling to 100000 frames in the simulated image time series, but we were not able to resolve changes in $\alpha$, and hence we were unable to detect variations in the blinking dynamics (data not shown).

The conclusions drawn from our results are important for fluorescence intermittency studies in different types of QDs or other fluorescent molecules with underlying power law blinking statistics. Many studies of fluorescence intermittency rely on the measurement of a time correlation function from intensity time traces of single molecules for use as a statistical tool to characterize the photophysical processes underlying the observed fluorescence dynamics. ${ }^{25,26}$ A single molecule approach is adopted for these studies due to its sensitivity to dynamic and population heterogeneities that are often masked by the averaging inherent in the ensemble approach. However, to characterize variations in blinking dynamics in QDs through correlation analysis with limited time sampling, a single molecule approach could not be used. Instead, measurements should be done on ensembles of QDs. We showed in Fig. 2 that the single dot time correlation functions of some of the QDs in the sample could be fitted by a three parameter power law. However, changes in the underlying blinking dynamics could not be resolved due to the large dot to dot variation in the recovered alpha exponent. Even for long imaging times, there exists a minimum lower bound on the size of the ensemble required to resolve variations. Power spectral measurement of blinking QDs has shown that ensemble measurements can provide the same

TABLE I. Summary of the mean fitting exponent $\alpha$ for the normalized intensity correlation functions of simulated image time series with different $m_{\text {on }}$ values and for different number of dots ranging from 800 to 25 . $m_{\text {off }}$ is set to 1.5. The number of blinking point emitters is included in brackets. Each $\alpha$ value is an average from 20 simulations. Uncertainties are standard deviations.

$\alpha$

(for different numbers of dots)

\begin{tabular}{ccccccc} 
& & & & & & \\
$m_{\text {on }}$ & $(800)$ & $(500)$ & $(300)$ & $(150)$ & $(50)$ & $0.40 \pm 0.1$ \\
\hline 1.5 & $0.43 \pm 0.04$ & $0.44 \pm 0.05$ & $0.47 \pm 0.08$ & $0.45 \pm 0.09$ & $0.40 \pm 0.10$ & $0.40 \pm 0.1$ \\
1.6 & $0.41 \pm 0.04$ & $0.39 \pm 0.04$ & $0.43 \pm 0.08$ & $0.38 \pm 0.09$ & $0.40 \pm 0.10$ & $0.40 \pm 0.1$ \\
1.7 & $0.35 \pm 0.03$ & $0.35 \pm 0.05$ & $0.33 \pm 0.05$ & $0.36 \pm 0.06$ & $0.40 \pm 0.10$ & $0.30 \pm 0.1$ \\
1.8 & $0.27 \pm 0.04$ & $0.27 \pm 0.04$ & $0.31 \pm 0.05$ & $0.25 \pm 0.06$ & $0.29 \pm 0.07$ & $0.29 \pm 0.08$ \\
1.9 & $0.2 \pm 0.04$ & $0.21 \pm 0.04$ & $0.22 \pm 0.05$ & $0.23 \pm 0.07$ & $0.24 \pm 0.06$ & 0.06 \\
\hline \hline
\end{tabular}


TABLE II. Summary of the mean fitting exponent $\alpha$ for the normalized intensity correlation functions of simulated image time series for an ensemble containing 150 point emitters with different $m_{\text {on }}$ values and for different number of frames ranging from 100 to $10000 . m_{\text {off }}$ is set to 1.5 . Each $\alpha$ value is an average from 20 simulations. Uncertainties are standard deviations.

\begin{tabular}{cccccc}
\hline \hline & \multicolumn{5}{c}{$\begin{array}{c}\alpha \text { for an ensemble of } 150 \text { dots } \\
\text { (for different numbers of frames) }\end{array}$} \\
\cline { 2 - 6 }$m_{\text {on }}$ & $(100)$ & $(500)$ & $(1000)$ & $(5000)$ & $(10000)$ \\
\hline 1.5 & $0.47 \pm 0.08$ & $0.41 \pm 0.08$ & $0.45 \pm 0.09$ & $0.41 \pm 0.08$ & $0.43 \pm 0.05$ \\
1.6 & $0.41 \pm 0.08$ & $0.40 \pm 0.08$ & $0.38 \pm 0.09$ & $0.40 \pm 0.06$ & $0.38 \pm 0.06$ \\
1.7 & $0.42 \pm 0.1$ & $0.35 \pm 0.05$ & $0.36 \pm 0.06$ & $0.34 \pm 0.06$ & $0.33 \pm 0.06$ \\
1.8 & $0.36 \pm 0.07$ & $0.31 \pm 0.09$ & $0.25 \pm 0.06$ & $0.25 \pm 0.07$ & $0.26 \pm 0.08$ \\
1.9 & $0.33 \pm 0.07$ & $0.26 \pm 0.06$ & $0.23 \pm 0.07$ & $0.18 \pm 0.07$ & $0.17 \pm 0.05$ \\
\hline \hline
\end{tabular}

information on blinking statistics as single dot measurements do. ${ }^{27}$ We further investigated the effect of temporal sampling and the number of point emitters in ensemble correlation measurements and demonstrated that the ability to resolve blinking distribution changes depends on the number of dots in the ensemble as well as the extent of temporal sampling.

Our simulation results support the conclusion that we were able to experimentally resolve differences in the underlying blinking statistics of the QDs via ICS measurement of the $\alpha$ exponent. The standard deviation on the $\alpha$ value decreases at higher laser intensities and the same trend is observed in simulations with large "on" distribution exponents. The results shown in Fig. 4 demonstrate that we can resolve changes in $\alpha$ as a function of laser power. Using the simulation results, we were able to confirm that these measurements were performed in a suitable sampling regime. The experimental results shown in Fig. 4 were obtained from systems containing approximately 100 emitting QDs in the analyzed area. Atomic force microscopy (AFM) measurements performed on the analyzed regions of the samples confirm that there are approximately 250 QDs within these areas, out of which, on average, 100 are fluorescently active as determined from the extracted fluorescence intensity traces (data not shown). The number of emitting QDs in Fig. 2 appears to be significantly less than 100 due to the significant fraction of QDs being in a dark state in the selected time series image frame.

In this study we measured systems with well separated (optically resolved) nanoparticles. The probability for a transition to a dark state in a QD is related to the distribution of trap states in its surroundings and the rate of formation of localized electron-hole pairs. ${ }^{28}$ The latter is dependent on the excitation intensity. We assume that the distribution of trap states around the spatially separated QDs is constant on average and use the variable excitation laser intensity as a tool to change underlying "on" time distribution. As our studies were conducted on systems where single QDs were optically resolved, we can be certain that our fluorescence measurements do not include contributions from resonance energy transfer or the absorption of energy from neighboring QDs due to recombination of high energy excitons. This density regime places a limit on the number of QDs that can be present in the sample. We can estimate 450 as the maximum number of QDs in a $64 \times 64$ pixels $^{2}$ area if we assume that fluorescence from a single dot spreads over an image area of $3 \times 3$ pixels $^{2}$. Direct application of the same analysis technique is possible for higher density samples where QDs are not fully spatially resolved as long as the QDs are not in very close proximity $(<10 \mathrm{~nm})$ of each other and is the subject of our future work.

\section{CONCLUSION}

In conclusion, we characterized the ensemble intensity time correlation function of blinking QDs using TIRF-ICS and computer simulation models. We show that the correlation function can be fitted to a three parameter power law. The change in the rate of the decay of the correlation function is a measure of the change in the underlying "on" time distribution. We found that detection of changes in the blinking distribution critically depend on adequate sampling and was most sensitive to the total number of emitters in the ensemble. We emphasize that the ensemble ICS method can successfully characterize blinking dynamics in the cases where temporal sampling is limited. Thus, it will be important to account for the blinking in future studies that employ ICS to measure mobility of QD labeled macromolecules in cellular systems. This study provides a basis for extending ICS mobility experiments to systems that employ QD labels where blinking fluctuations will be present.

Based on this work, we expect to be able to measure changes in the blinking of QDs as a function of other environmental parameters apart from laser intensity. It has been previously shown that, due to their intrinsic sensitivity to the surrounding chemical environment, modifications of the surface interface of nanocrystals significantly affect their photophysical properties. ${ }^{19,29}$ Moreover, recent reports suggest that reducing agents affect quantum dot blinking, ${ }^{30}$ further suggesting that this process may be modulated and exploited for the development of sensing technologies. Thus, accurate characterization of the variation in the blinking dynamics under controlled experimental conditions is of particular relevance to future applications utilizing fluorescence of QD nanoparticles for sensing purposes.

\section{ACKNOWLEDGMENTS}

We would like to thank Dr. D. Ronis, Dr. S. Costantino, and Dr. K. Heinze for helpful discussions and suggestions. 
This project was financially supported by grants from CIHR (Neurophysics training grant), NSERC, CFI, FQRNT, RQMP, and HFSP.

${ }^{1}$ M. A. Hines and P. Guyot-Sionnest, J. Phys. Chem. 100, 468 (1996).

${ }^{2}$ M. Nirmal and L. Brus, Acc. Chem. Res. 32, 407 (1999).

${ }^{3}$ D. J. Norris and M. G. Bawendi, Phys. Rev. B 53, 24 (1996).

${ }^{4}$ X. Michalet et al., Science 307, 538 (2005).

${ }^{5}$ M. Bruchez, M. Moronne, P. Gin, S. Weiss, and A. P. Alivisatos, Science 281, 2013 (1998).

${ }^{6}$ W. C. Chan and S. Nie, Science 281, 2016 (1998).

${ }^{7}$ M. Nirmal, B. O. Dabbousi, M. G. Bawendi, J. J. Macklin, J. K. Trautman, T. D. Harris, and L. E. Brus, Nature (London) 383, 802 (1996).

${ }^{8}$ M. Dahan, S. Levi, C. Luccardini, P. Rostaing, B. Riveau, and A. Triller, Science 302, 442 (2003).

${ }^{9}$ S. Doose, J. M. Tsay, F. Pinaud, and S. Weiss, Anal. Chem. 77, 2235 (2005).

${ }^{10}$ J. Widengren, U. Mets, and R. Rigler, J. Phys. Chem. 99, 13368 (1995)

${ }^{11}$ P. Schwille, S. Kummer, A. A. Heikal, W. E. Moerner, and W. W. Webb, Proc. Natl. Acad. Sci. U.S.A. 97, 151 (2000).

${ }^{12}$ M. Kuno, D. P. Fromm, H. F. Hamann, A. Gallagher, and D. J. Nesbitt, J. Chem. Phys. 112, 3117 (2000).

${ }^{13}$ M. Kuno, D. P. Fromm, H. F. Hamann, A. Gallagher, and D. J. Nesbitt, J. Chem. Phys. 115, 1028 (2001).

${ }^{14}$ G. Margolin and E. Barkai, J. Chem. Phys. 121, 1566 (2004).

${ }^{15}$ G. Margolin and E. Barkai, Phys. Rev. Lett. 94, 080601 (2005); M. K. Kuno, D. P. Fromm, H. F. Hamann, A. Gallagher, and D. J. Nesbitt, J.
Chem. Phys. 115, 1028 (2001).

${ }^{16}$ M. F. Paige, E. J. Bjerlend, and W. E. Moerner, Single Mol. 2, 191 (2001).

${ }^{17}$ W. H. Press, B. P. Flannery, S. A. Teukolsky, and W. T. Vetterling, Numerical Recipes in C: The Art of Scientific Computing, 2nd ed. (Cambridge University Press, Cambridge, England, 1993).

${ }^{18}$ W. G. J. H. M. van Sark, P. L. T. M. Frederix, A. A. Bol, H. G. Gerritsen, and A. Meijerink, ChemPhysChem 3, 871 (2002).

${ }^{19}$ R. Verberk, J. W. M. Chon, M. Gu, and M. Orrit, Physica E (Amsterdam) 26, 19 (2005).

${ }^{20}$ P. W. Wiseman, J. A. Squier, M. H. Ellisman, and K. R. Wilson, J. Microsc. 200, 14 (2000)

${ }^{21}$ G. Messin, J. P. Hermier, E. Giacobino, P. Desbiolles, and M. Dahan, Opt. Lett. 26, 1891 (2001).

${ }^{22}$ K. T. Shimizu, R. G. Neuhauser, C. A. Leatherdale, S. A. Empedocles, W. K. Woo, and M. G. Bawendi, Phys. Rev. B 63, 205316 (2001).

${ }^{23}$ R. Verberk and M. Orrit, J. Chem. Phys. 119, 2214 (2003).

${ }^{24}$ A. Y. Kobitski, C. D. Heyes, and G. U. Nienhaus, Appl. Surf. Sci. 234, 86 (2004).

${ }^{25}$ K. D. Weston, P. J. Carson, H. Metiu, and S. K. Buratto, J. Chem. Phys. 109, 7474 (1998).

${ }^{26}$ R. M. Dickson, A. B. Cubitt, R. Y. Tsien, and W. E. Moerner, Nature (London) 388, 355 (1997).

${ }^{27}$ M. Pelton, D. M. Grier, and P. Guyot-Sionnest, Appl. Phys. Lett. 85, 819 (2004).

${ }^{28}$ I. S. Osad'ko, JETP Lett. 79, 416 (2004).

${ }^{29}$ S. R. Cordero, P. J. Carson, R. A. Estabrook, G. F. Strouse, and S. K. Buratto, J. Phys. Chem. B 104, 12137 (2000).

${ }^{30}$ S. Hohng and T. Ha, J. Am. Chem. Soc. 126, 1324 (2004). 